\title{
THE VASCULAR RESPONSE IN CHRONIC RHEUMATOID ARTHRITIS
}

\author{
BY
}

\author{
A. BENATT AND H. J. TAYLOR * \\ From the St. John Clinic, London, S.W.1
}

Received April 21, 1940

It has been suggested that a deficient blood supply might bring about abnormalities in the joints, just as it can in other tissues, and that vascular disturbances might be an ætiological factor in the causation of rheumatoid arthritis and osteo-arthritis. Savage and Taylor (1939) by means of oxygen and carbon dioxide measurements in the joints have shown that in certain cases of these diseases such a deficiency may exist. Goldhaft, Wright, and Pemberton (1933) successfully repeated the work of Wollenberg (1909) who showed that ligation of the patellar blood vessels in a dog produced symptoms of hypertrophic arthritis. The relationship between lack of blood supply and osteoarthritis is illustrated by the following case seen by one of us. A man of 59 years of age suffered for five years from pains in the left knee and hip, the area round the latter being dead cold to the touch. The X-ray examination showed marked osteo-arthritis in the left hip, the presence of some unabsorbed material on this side, and distinct signs of arteriosclerosis in the left lower thigh. The patient stated that the only injections he had received were of gold into both buttocks two years ago. The non-absorption of this material, together with arteriosclerosis and coldness, on the affected side only, pointed to a deficient blood supply.

Many rheumatic subjects are very sensitive to cold weather and complain of cold hands and feet, and this has led to many investigations on skin capillaries. Deviations from the normal have been described, such as constriction (Lunedei and Coradini, 1930, and Bisset and Woodmansey, 1932); decrease in the number of capillaries above joints, where there were Heberden's nodes, and where the capillaries were also constricted (Kovacs, Wright, and Duryee, 1933): these authors saw tortuosity of the small skin vessels in 60 per cent of cases of osteoarthritis in comparison with 35 per cent in respect of rheumatoid arthritis, but they attribute this to the higher age of the former; they conclude that many changes from normal do occur in the skin capillaries and skin temperature, but owing to the lack of consistency of the observations they can not be considered as responsible for the pathological changes. Kersley (1937) agrees with this view.

The problem therefore is whether, apart from obstructed blood flow to the

\footnotetext{
* Holder of an Alexander McLean Fellowship of the Empire Rheumatism Council.
} 
joints, there is any functional vascular abnormality that leads to changes in the joints in cases of rheumatoid arthritis of unknown origin. Disturbance in the vascular constrictor tone is likely to be suspected, as it is met with in the so-called Raynaud syndrome and in acrocyanosis. In both these maladies the arteriolar constrictor tone is increased and in acrocyanosis there is also atonia of the subpapillary venous plexuses of the skin. Patients suffering from these vascular disorders are, like rheumatic subjects, very susceptible to cold. If in rheumatic diseases abnormal tone is present, one would expect a reaction to heat and cold similar to that in Raynaud's syndrome or in acrocyanosis. A suitable means to test such a vascular response is by the use of contrast baths.

The normal reaction of the blood vessels to this type of bath has been determined by Uprus, Gaylor, and Carmichael (1936). Our experiments are based on the fact observed by Gibbon and Landis (1932) that immersing one forearm in hot water produced vasodilatation in the other arm as well. More particularly Woodmansey, Collins, and Ernst (1938) have studied this reaction in rheumatic subjects. The times of immersion which these authors employed were too short for any sufficient vascular response.

\section{METHOD}

The usual method of finding if the capillaries are dilated or constricted is by measurements of the skin temperature. We used the nail bed of the index or third finger. According to Lewis and Pickering (1933) this shows the earliest response when the arm is exposed to hot or cold air, owing to the presence of numerous arterio-venous anastomoses at the finger tips (Grant and Bland, 1931). Gibbon and Landis (1932) have confirmed such response when the arm is heated with warm water.

Skin temperature measurements were carried out by us using the well known thermocouple-galvanometer method. The room was kept at as constant a temperature as possible during an experiment, but it varied somewhat from day to day from $18^{\circ}$ to $21^{\circ} \mathrm{C}$. The subject was seated comfortably in a chair so that the arms were able to relax. The two thermocouples were fixed one on each hand, usually at the nail bed of the index, but sometimes of the third finger. Measurements were carried out at intervals, until the temperature of the digits showed a constant figure, usually after 10-20 minutes, but exceptionally after 40 minutes. One arm was then immersed in a large bowl containing warm or cold water, as the case might be. The temperature of the warm water was kept constant by an immersion heater. The temperature of the cold water changed very little in the short time (8-12 minutes) during which the arm was immersed. The amount of water was sufficient to cover the arm from the middle of the hand up to the middle of the biceps. The finger to which the thermocouple was attached remained well above the surface of the water. Temperature readings were noted at intervals of half a minute to two minutes, from the digits of the immersed and non-immersed arms. When satisfactory readings had been taken of the response to heat or cold, as the case might be, the hot water was changed for cold or vice versa. This process was repeated several times. It was not found possible to measure the rectal temper- 
ature during an experiment, and we had to be satisfied with mouth temperatures. This invariably rose by $0.4^{\circ}-0.5^{\circ} \mathrm{C}$. when the arm was immersed in hot water, and fell $\left(0 \cdot 2^{\circ}-0 \cdot 3^{\circ} \mathrm{C}\right.$. $)$ when the arm was immersed in cold water, a point which has already been discussed by other writers.

Observations on the Reaction to Contrast Baths

These have been carried out on the following groups:-

(A) Normal subjects (10 cases)

(B) Non-arthritic subjects with Raynaud's syndrome in various stages (such as when cold- or warm-handed) (8 cases)

(C) Non-arthritic subjects with acrocyanosis (5 cases)

(D) Subjects with rheumatoid arthritis-

(a) with a normal blood sedimentation rate (21 cases) ;

(b) with a raised blood sedimentation rate ( 9 cases).

We have selected from each group cases that showed a typical reaction.

\section{(A) Normal subjects}

The main features of the response of a normal subject to contrast baths are shown in Fig. 1.

(a) In cold water there is an immediate rapid fall in the temperature of the fingers of both the immersed and non-immersed arms.

(b) In hot water this fall continues for a short period (5-8 minutes) and is followed by a rapid rise of both fingers.

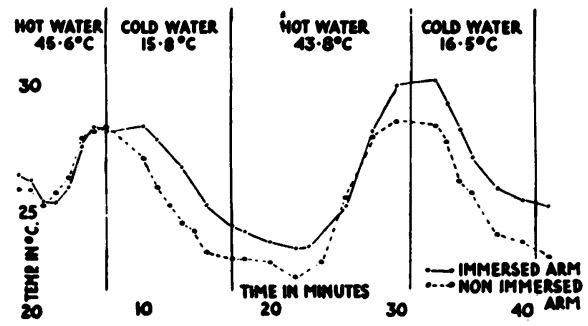

Fis. 1.-Normal subject, aged 38. In hot water there is a short fall in temperature, followed by a rapid rise in the digits of both the immersed and non-immersed arms. In cold water a rapid fall in temperature of both sides is shown. On immersion again in hot water there is a rapid rise in temperature after a delay of 6 minutes. After this cold water produces the usual rapid fall.

We found that if the initial skin temperature was high, that is if the arm was already in a state of vascular dilatation, then immersion in hot water could only produce a slight increase in this state.

\section{(B) Non-Arthritic subjects with Raynaud's Syndrome}

In general, patients suffering from this vascular disorder showed when they were warm-handed a normal or nearly normal response. When they were cold-handed the response was greatly delayed; but, once established, was normal in character. Variations occurred both from subject to subject and in the same subject at different times. 
Observations on a typical case were as follows:

A woman, aged 33, had suffered for several years from cold arms, hands, and feet. Two years before sympathectomy had been performed on one leg without any effect on the Raynaud's syndrome. In particular the left hand and arm felt very cold, and the little finger showed marked cyanosis. Sometimes, however, the temperature of the hands was quite normal. There were no symptoms of arthritis or of heart failure.

We found variable reactions such as the following :

1. A complete lack of response to both heat and cold in both hands. The initial temperature of the digits was low $\left(20 \cdot 8^{\circ} \mathrm{C}\right.$. on the immersed, $20 \cdot 3^{\circ} \mathrm{C}$. on the non-immersed side). Cold water at $14^{\circ} \mathrm{C}$. caused a drop in temperature on the immersed side to $17 \cdot 9^{\circ} \mathrm{C}$. in 10 minutes. On immersion in hot water at $45 \cdot 5^{\circ} \mathrm{C}$. no rise occurred even after 20 minutes' immersion. Subsequent immersion in hot or cold water could not release the vaso-constriction (see Fig. 2).

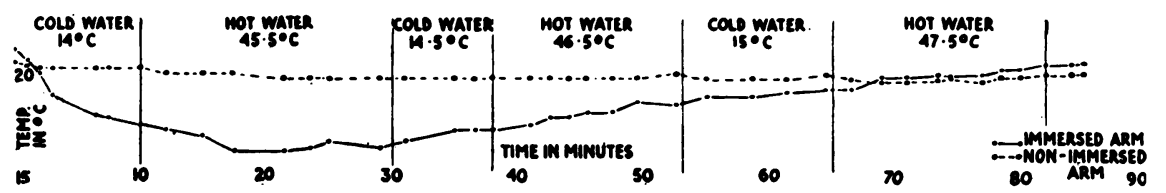

Fig. 2.-Subject suffering from Raynaud's syndrome. When cold-handed, practically no response in either side occurs, though at first the immersed hand becomes a little colder.

2. No response for a long time on the immersed side (initial temperature $20 \cdot 4^{\circ} \mathrm{C}$.) either in hot or cold, whilst the non-immersed arm (initial temperature $23.4^{\circ} \mathrm{C}$.) had the paradoxical response of a rise in cold water at the beginning (see Fig. 3).

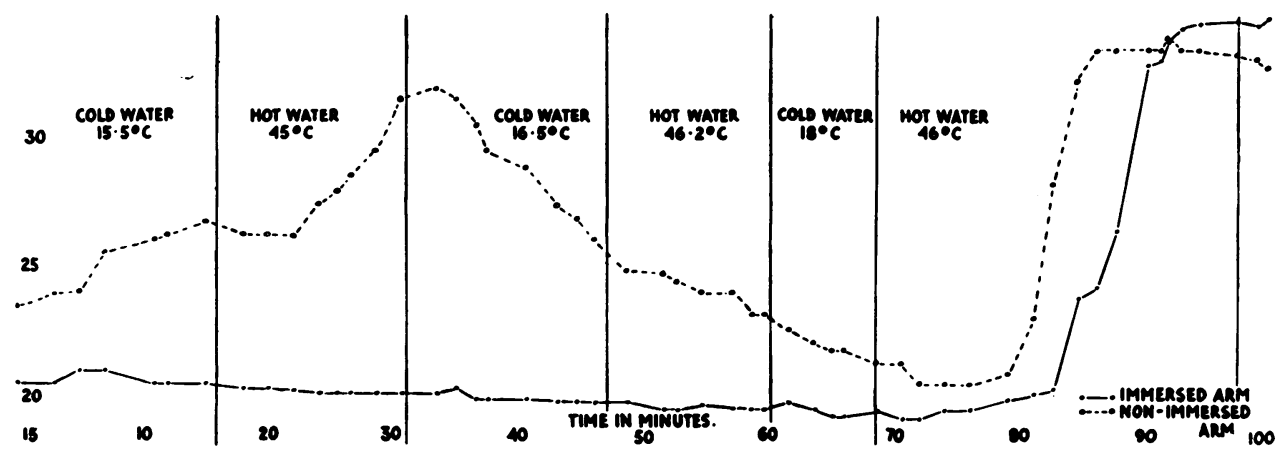

FIG. 3.- Subject suffering from Raynaud's syndrome. Immersed arm shows no reaction to heat or cold for a long time. A paradoxical rise in the temperature of the non-immersed arm to the stimulus of cold is shown; as also is the rapid response on both sides when this rise has gradually passed off.

3. When the same subject was investigated at a time that he was free from symptoms and warm-handed, an almost normal response was obtained on the immersed side, while the non-immersed side, after reacting poorly to cold, showed a normal response. 
(C) Non-Arthritic subjects with Acrocyanosis

In general, subjects suffering from this disorder can show an almost normal reaction even when cold-handed; it is, however, characterized by the suddenness of the response to heat. On the other hand, after immersion in cold water, the vaso-constriction may become so intense that a response similar to Raynaud's syndrome when cold-handed is obtained. When response was established either to heat or cold, the graph representing the reaction did not proceed smoothly but showed an unexplained jerkiness.

The following case may be taken as typical: A woman, aged 27, had suffered from acrocyanosis in the hands, feet, and lower part of the legs for many years. There were no signs of disturbance in the endocrine glands nor of heart failure. Both hands felt cold to the touch and had a bluish-red colour. There were no signs of arthritis.

1. Sometimes there was a reaction that resembled the normal.

2. In another observation hot water produced a rise in temperature of both digits similar to a normal reaction, but more rapid. When immersed in cold water again there was a rapid fall in temperature which continued, when the arm was again immersed in hot water, for a long period, and no rise in temperature occurred (see Fig. 4).

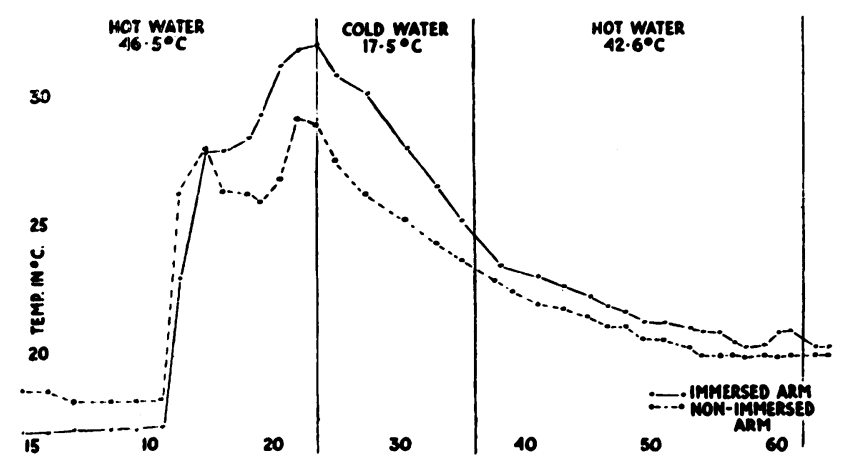

Fig. 4.- Subject suffering from acrocyanosis. The sudden rise in temperature of both sides follows the usual initial delay in hot water. Cold water produces an immediate fall in temperature, which continues on subsequent immersion in hot water, the latter failing to release the vaso-constriction even after 26 minutes' immersion.

\section{(D) Subjects suffering from Rheumatoid Arthritis}

(a) With a normal blood sedimentation rate (21 cases)

These subjects gave a typically normal response. Many observations were carried out at various times on each of these subjects in addition to many others, and all of them showed a normal reaction.

The following may be given as an example: A woman, aged 48, had hysterectomy performed 15 years ago, having previously had three normal births. B.P. was $170 / 110 \mathrm{~mm}$. She had suffered from painful swelling in the right knee for three years. No marked bony changes were shown on X-ray examination. Blood sedimentation rate 5 (Westergreen method, 1 hour). The illustration has not been reproduced as it was similar to Figs. 1 and 5. 
(b) With a raised blood sedimentation rate ( 9 cases)

The results obtained may be given under the following headings:

1. Rheumatoid arthritis, without any abnormalities of posture, showed a normal response to heat and cold.

2. Rheumatoid arthritis, characterized by a long history of infectious diseases and with marked anatomical changes in the joints associated with postural defects, gave a sluggish response at each observation.

The following cases may be given as examples :-

A woman, aged 58, had suffered from rheumatism after her first pregnancy. For some years she had pains in her finger joints and wrists with severe recurrent swelling and pain. On X-ray examination destructive changes in some metacarpo-phalangeal joints. The blood sedimentation rate was between 14 and 19 .

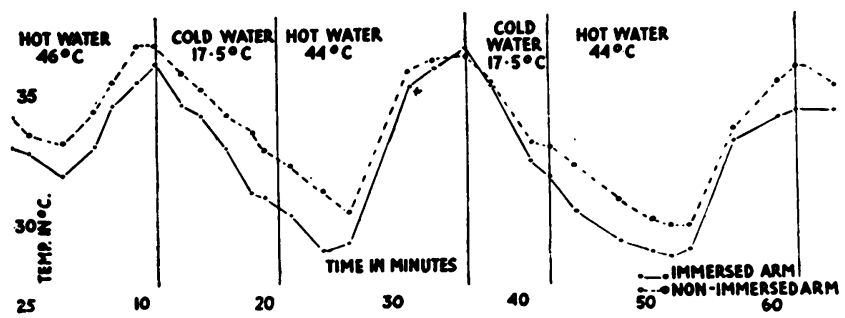

FIG. 5.-Subject with rheumatoid arthritis (raised blood sedimentation rate). A typically normal response.

A woman, aged 28 , had suffered from many infections in childhood, but not from rheumatic fever. After scarlet fever rheumatic pains started with swelling in the hands, ankles, and elbows, and with iridocyclitis of the left eye. Her general condition was poor and she could not walk without orthopædic support because of severe deformities in both hands and feet. X-ray examination of the hands showed chronic rheumatoid arthritis with bony fusion of the carpal bones and ankylosis of several phalangeal joints. She had very pronounced deformities of the hands and arms. Her blood sedimentation rate was 40 . There was a sluggish rise in temperature on the immersed side, while the non-immersed side showed a normal response (Fig. 6).

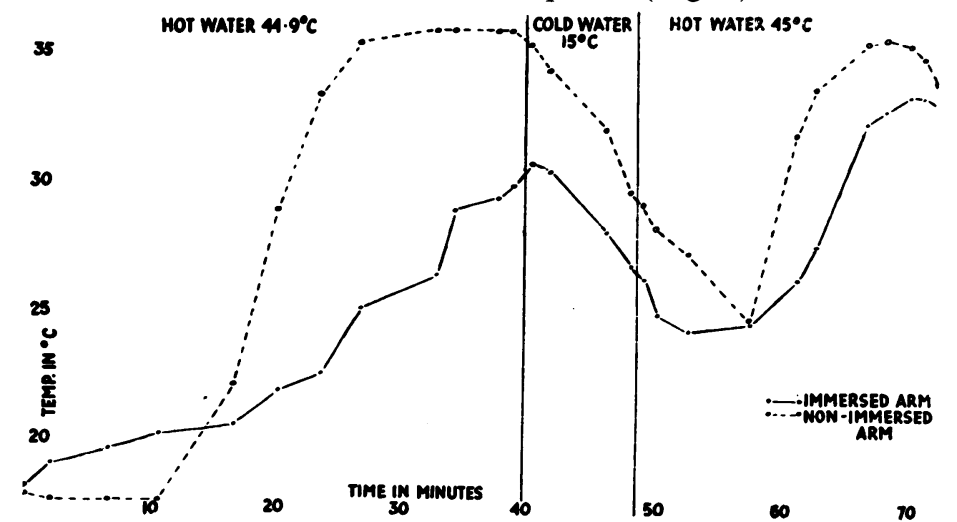

FIG. 6.-Subject with rheumatoid arthritis and postural deformities (raised blood sedimentation rate). A sluggish rise in temperature on the immersed side occurs while the non-immersed shows a normal response. 
Discussion

The general features of the response of normal subjects to heat and cold closely resemble those obtained by Lewis and Pickering (1931-33), by Uprus, Gaylor, and Carmichael (1936), and by Gibbon and Landis (1932), although the actual limbs used and the procedure differed somewhat. Cooling one arm produces an immediate fall in temperature; heating one arm produces a delayed rise in temperature of both the immersed and non-immersed arm lasting from 8 to 10 minutes. Carmichael et al. (1936) pointed out that two factors are responsible for the cooling effect; the sensory stimulus producing vasoconstriction, and the cooling of the blood. The stimulus for the vascular dilatation is the rise in blood temperature; this must overcome the vasoconstrictor tone which may vary from subject to subject. When a normal subject was investigated, at one time with warm, and at another time with cold hands, the heat response under the latter conditions showed a delay which did not, however, exceed 8-10 minutes. Lewis and Pickering (1931-33) have shown that dilatation begins in the warmer fingers; the delay in response of the cooler fingers is attributed to the direct constrictor effect of cold on the arterioles. In cases of so-called Raynaud's syndrome with cold hands, when an increased vaso-constrictor tone is known to be present, not only is the time required to release the vaso-constriction lengthened, but the characteristics of such a response in the immersed and non-immersed limbs may be entirely different. In this vascular disorder we noted three principal types of reactions:

(a) the vaso-constrictor tone may remain unchanged in both arms;

(b) the vaso-constrictor tone may be released in one arm only, whilst the other side remains constricted;

(c) a paradoxical rise in cold and a fall in hot water may occur.

Once the reaction is initiated on both sides, it continues to be normal in type, as Lewis and Pickering have already pointed out. In acrocyanosis a rapid heat response may be obtained. However, after immersion of one limb in cold water the vaso-constrictor effect produced may be so great that subsequent immersion in hot water fails to release it.

In our subjects with rheumatoid arthritis of various types we have found no similarity, in their reaction to contrast baths, to these fundamental types of functional vascular disturbance. Rheumatoid arthritis and Raynaud's disease are only occasionally associated, whereas one would expect this more frequently if functional vascular disturbance were an ætiological factor in arthritis. One case of Raynaud's syndrome in which arthritic changes in the terminal joints of the finger were observed has been described by Lewis (1937); the changes were considered to be due to old re-canalized thrombi which severely restricted the blood flow to these joints-an anatomical cause for the condition.

The reaction of our arthritic subjects was tested on many different occasions in order to detect a possible temporary disturbance of vascular function; but even when the temperature of the hands was low, a quite normal response was obtained. Nor was there any sign of vaso-constriction that could not be released, such as occurs in acrocyanosis. The delay in heat response in these cases after immersion in cold water did not exceed the time observed in normal 
subjects. A distinct delay in heat reaction was, however, noted in a severe case of rheumatoid arthritis of long standing, high sedimentation rate, and marked deformities of the arms and hands. Other subjects with all the same changes except the postural abnormalities gave a normal reaction. It has been found out by Uprus, Gaylor, Williams, and Carmichael (1935) that limbs with an abnormality in posture show a pronounced delay in heat response, which could also be elicited from a normal subject who imitated this abnormal posture. We are justified, therefore, in attributing the result in rheumatoid arthritis to this cause.

We conclude, that as far as the vascular system is concerned in the ætiology of rheumatoid arthritis and osteo-arthritis, a permanent arrest of blood flow caused by thrombi, by severe arteriosclerosis, or by any mechanical means, may produce joint and bony changes. In cases of vascular disorders like Raynaud's syndrome when associated with arthritis one has to consider the possibility of obstruction of the vessels leading to the affected area. No specific nor particular type of functional vascular reaction, as tested in contrast baths, could, however, be elicited in the various forms or stages of rheumatoid arthritis that we were able to investigate. This does not exclude the fact that many rheumatic patients are especially sensitive to cold, but this cannot be ascribed to abnormal yascular constrictor tone.

\section{SUMMARY}

1. Arthritic changes have been seen in cases in which the blood supply to a joint has been restricted.

2. Investigations have been carried out to find if there might be any functional vascular disturbance in various types and stages of chronic rheumatoid arthritis. The reaction of rheumatic subjects to contrast baths has been compared with that of normal subjects and of subjects suffering from increased vasoconstrictor tone such as Raynaud's syndrome or acrocyanosis.

3. Unless a marked postural deformity was present all rheumatic subjects showed a reaction well within normal limits.

4. Several types of reaction to contrast baths were obtained in cases of socalled Raynaud's syndrome and acrocyanosis. There was no similarity to the reactions obtained in cases of rheumatoid arthritis.

\section{REFERENCES}

Bisset, A., and Woodmansey (1932). Lancet, 2, 620.

Gibbon, J. H., and Landis, E. N. (1932). J. clin. Invest., 11, 1019.

Goldhaft, A., Wright, L. M., and Pemberton, R. (1933). Ann. intern. Med., 6, 1591.

Grant, R. T., and Bland, E. F. (1929-31). Heart, 15, 385.

Kersley, J. D. (1937). Acta. rheum., 9, 2.

Kovacs, J., Wright, J. S., and Duryee, A. W. (1933). J. Amer. med. Ass., 100, 1018.

Lewis, T. (1937). J. clin. Sci., 3, 287.

Lewis, T., and Pickering, G. W. (1931-33). Heart, 16, 33.

Lunedei, A., and Coradini, C. (1930). Acta. rheum., $2,2$.

Pemberton, R. (1935). Arthritis and Rheumatic Conditions. Philadelphia.

Savage, O., and Taylor, H. J. (1939). Reports of Empire Rheumatism Council. March 1939.

Uprus, V., Gaylor, G. B., Williams, D. J., and Carmichael, E. A. (1935). Brain, 58, 448.

Uprus, V., Gaylor, G. B., and Carmichael, E. A. (1936). J. clin. Sci., 2, 301.

Wollenberg, J. (1909). Cited by Pemberton R. (1930). Acta. rheum., 6, 11.

Woodmansey, A., Collins, D. H., and Ernst, M. M. (1938). I.ancet, 2, 1350. 